\title{
Monte Carlo tests of small-world architecture for coarse-grained networks of the United States railroad and highway transportation systems
}

Preston R. Aldrich ${ }^{1, *}$, Jermeen El-Zabet ${ }^{1}$, Seerat Hassan ${ }^{1}$, Joseph Briguglio ${ }^{1}$, Enela Aliaj ${ }^{1}$, Maria

Radcliffe $^{1}$, Taha Mirza ${ }^{1}$, Timothy Comar ${ }^{2}$, Jeremy Nadolski ${ }^{2}$, Cynthia D. Huebner ${ }^{3}$

${ }^{1}$ Department of Biological Sciences, Benedictine University, Lisle, IL, USA

${ }^{2}$ Department of Mathematical and Computational Sciences, Benedictine University, Lisle, IL, USA

${ }^{3}$ Northern Research Station, USDA Forest Service, Morgantown, WV, USA

*Corresponding author. Email: paldrich@ ben.edu 
Abstract. Several studies have shown that human transportation networks exhibit small-world structure, meaning they have high local clustering and are easily traversed. However, some have concluded this without statistical evaluations, and others have compared observed structure to globally random rather than planar models. Here, we use Monte Carlo randomizations to test U.S. transportation infrastructure data for small-worldness. Coarse-grained network models were generated from GIS data wherein nodes represent the 3,105 contiguous U.S. counties and weighted edges represent the number of highway or railroad links between counties; thus, we focus on linkage topologies and not geodesic distances. We compared railroad and highway transportation networks with a simple planar network based on county edge-sharing, and with networks that were globally randomized and those that were randomized while preserving their planarity. We conclude that terrestrial transportation networks have small-world architecture, as it is classically defined relative to global randomizations. However, this topological structure is sufficiently explained by the planarity of the graphs, and in fact the topological patterns established by the transportation links actually serve to reduce the amount of small-world structure.

Keywords highway, network, railroad, small-world, transportation. 


\section{Introduction}

Human transportation systems are vital components of a nation's economy, promoting the movement and delivery of persons, goods, and services (Rodrigue et al., 2013). There are also collateral movements of undesirable factors such as chemical toxins (Kara and Verter, 2004), human diseases (Morris, 2004), and invasive species (Floerl et al., 2009; Harrison et al., 2002; Hulme, 2009; Meyerson and Mooney, 2007; Perrings et al., 2005), each carrying a cost. For example, it is estimated that invading alien species bring economic losses of nearly $\$ 120$ billion per year in the United States (Pimentel et al., 2005). Partly because the stakes are high in this new era of globalism, there is considerable interest in understanding how easily things move long distances through a transportation system, and the extent to which the infrastructure promotes local contagion and mixing.

Networks are useful for the study of transportation systems. The mathematical field of graph theory, which treats networks as mathematical objects, is considered to have arisen in 1735 with Euler's classic transportation problem involving the Konigsberg bridges (Newman, 2010). Since then, an extensive literature has accumulated regarding the organization of human terrestrial transportation networks, reviewed in Blanchard and Volchenkov (2008), Barthélemy (2011), Rodrigue et al. (2013), and Lin and Ban (2013). Many transportation networks seem to share some basic topological features. Some of this structure is thought to emerge as a balance between minimizing the costs of constructing and maintaining the transportation systems versus the ease of traversing them (Gastner and Newman, 2006; Latora and Marchiori, 2003; Valverde et al., 2002). For example, many studies have concluded that terrestrial transportation networks display a small-world architecture: urban street networks (Jiang and Claramunt, 2004) urban public transportation networks (Latora and Marchiori, 2002, 2003; Sienkiewicz and Hołyst, 2005), and railroads (Li and Cai, 2007; Seaton and Hackett, 2004; Sen et al., 2003; Wang et al., 2008).

The small-world phenomenon was first characterized for a social system (Travers et al., 1969) where it was shown that U.S. acquaintance networks linked people together in roughly six steps. This was 
a remarkable finding given the large size of the U.S. population and the fine-scale complexity of social relationships. The random graph theory of Erdős and Rényi (Erdős and Rényi, 1959, 1960, 1961) had already shown that large random networks were easily traversed by virtue of shortcuts through the network, especially compared to regular networks such as grids or lattices. Several decades later, physicists Watts and Strogatz (1998) recognized the ubiquity of small-world networks and formalized their quantitative analysis. They showed that many naturally occurring complex networks share features of both regular graphs (high local organization) and random graphs (easily traversed), this by virtue of a small number of random re-wirings that introduce shortcuts across an otherwise highly organized network. They introduced a measure of fine-scale structure, where the clustering coefficient of node $x$ $\left(C_{x}\right)$ is the probability that nodes $y$ and $z$ also are linked given that they both link to node $x$. The average clustering coefficient of a small-world network should be much greater than that of a random network. They measured the ease of traversability by the characteristic path length $(L)$ which is the average of the all the shortest path distances between node pairs in a network. If small-world networks are as easily traversed as random graphs, Erdős and Rényi theory (Erdős and Rényi, 1959, 1960, 1961) indicated that path lengths should scale with the $\log$ of the graph size $(L=\log (N) / \log (\mathrm{z}))$, meaning that even large increases in the number of nodes leads to only modest increases in the average number of links separating nodes.

The success of the small-world network model is indisputable, having been identified in complex systems ranging from actor networks, brain neural networks, to cellular networks. It has been less clear, however, exactly how best to judge if a network displays significant small-world structure. The Watts and Strogatz (1998) small-world model was defined by its boundary conditions, regular and random graphs. But it is worth asking, are these the correct boundary conditions for the analysis of all complex networks? Some have suggested that Erdős-Rényi random graphs, i.e., those that are globally randomized (here, ER graphs), are not always the best models for testing structure in real networks (Cardillo et al., 2006; Newman, 2003). For one thing, ER random graphs lack clustering; the probability that two randomlychosen nodes are linked and complete a triangular relationship is simply the probability $p$ that they are 
linked, so the expectation of the clustering coefficient $C=p$. So just how much clustering must one see in order to judge that a real network has small-world structure? The threshold is rather low relative to ER random graphs. Moreover, it is worth asking what is being tested when we compare an ER random graph to a terrestrial transportation network, for example, which is for the most part a planar graph. The ER random graph would include edges between spatially disjunct nodes that could not possibly form links in the real world (e.g., a direct link between cities on opposite coasts of the United States without any intervening intersections). Then does a significant finding indicate something about transportation networks in particular, or does it simply highlight the differences between spatially constrained planar graphs versus unconstrained ER random graphs?

The literature is quite varied when it comes to statistical tests of small-worldness in terrestrial transportation networks, but all indicate small-world structure, reviewed in Xu and Sui (2007). Some studies have concluded small-world structure based mainly on the observation of high clustering and small diameter or characteristic path length (Li and Cai, 2007; Sienkiewicz and Hołyst, 2005). Many compare the observed structure to the theoretical expectations for an ER globally random graph as outlined by Watts-Strogatz (Seaton and Hackett, 2004; Sen et al., 2003). Few studies compare transportation networks to random planar graphs. Masucci et al. (2009) examined the London street network and made a random planar graph with as many nodes but distributed as a Poisson process and randomly linked; this single random model was then compared to the real network. Cardillo et al. (2006) compared observed planar graphs with randomly-generated planar graphs of two types: minimum spanning trees and greedy triangulations. And some have developed or borrowed alternative but related metrics to quantify small-worldness including network efficiency (Latora and Marchiori, 2003) and spatial autocorrelation methods (Xu and Sui, 2007).

A less-explored approach would be to compare the observed transportation network to multiple spatially constrained, planar randomizations of the same network. One study of the U.S. highway system (Kalapala et al., 2006) generated such randomizations, but this was for assessing scale-free structure, not small-world structure. Planar randomizations can be performed through a Monte Carlo simulation 
(Metropolis and Ulam, 1949) by allowing random edge formation only between spatially adjacent nodal entities, such as between counties or states that share a border. In research on brain networks (Humphries et al., 2006), Monte Carlo randomizations were used to reveal small-world structure in the organization of linkages between neuronal sectors in the brain. They introduced the small-world coefficient, $S$, which represents a balance between the clustering and the characteristic path length, each normalized by the mean of the metric calculated across the Monte Carlo replicates $\left(C_{R}\right.$ and $L_{R}$, respectively). Thus, $S=$ $\left(C / C_{R}\right) /\left(L / L_{R}\right)$, and small-world networks should exhibit a profile where $C / C_{R}>1.0$ and $S>1.0$.

In the present study, we examine the network structure of the United States railroad and highway transportation systems. We coarse-grained GIS data into networks where nodes represent counties and weighted edges represent the number of railroad or highway crossings between counties. Coarse-graining is a procedure whereby nodes are merged with other adjacent nodes in a network, and the newly-formed nodes inherit the links of the former nodes (Kim, 2004; Song et al., 2005); this is also referred to as forming a contraction of the original graph (Nishizeki et al., 2008). For example, in a study of the railroad network in China (Wang et al., 2008), a 'space L' intersection network was constructed where each of 3,110 train stations were treated as nodes, and two nodes were linked if a train passed between the stations. This topology was then compared to a coarse-grained 'space G' network in which the 3,110 train stations were merged by city yielding 298 city nodes with edge weights representing the number of rail links running between cities. They found that although the coarse-grained network (space G) was smaller than the intersection network (space L), both network types displayed the same key topological features, including small-world structure.

Our principle contributions to transportation network research are two-fold. (1) We provide direct comparisons between a railroad network, a highway network, and a simple planar network in which links are defined by county adjacency and not by transportation tracks, all at the national level. Many of the existing studies are nicely replicated, though the scale of the observation is very often at the city-level, not the nation. Of the national-level studies that exist, such as for India (Sen et al., 2003), Poland (Sienkiewicz and Hołyst, 2005), the United States (Kalapala et al., 2006), and China (Wang et al., 2008), 
in each case they examined either highways (roads) or railroads, but not both. Moreover, not all of these studies tested for small-world structure. (2) We apply Monte Carlo randomization procedures to test for significant structural differences using the small-world coefficient, which has not been done previously for terrestrial transportation networks. In particular, we examine significance under two regimes, global Erdős-Rényi (ER) randomization and planar randomization. We also compare the results of the transportation network tests with those for an observed, non-transportation planar network of the same size. This provides some indication as to whether significant topological structure associates with particular types of transportation networks as opposed to being a general feature of planar graphs.

\section{Materials and Methods}

\subsection{Transportation infrastructure data}

Geographic Information Systems (GIS) data were obtained for 2011 from the U.S. Department of Transportation (US DOT, 2011) through the NTAD-2011 database (National Transportation Atlas Data2011: Bureau of Transportation Statistics, Research and Innovative Technology Administration (RITA), US Department of Transportation). Three raw data files were downloaded: National Highway Planning Network (polyline; Federal Highway Administration), Railway Network (polyline; Federal Railroad Administration), and U.S. County Boundaries (polygons, TIGER/Line Shapefile, U.S. Census Bureau).

\subsection{Network construction}

Data files were opened and reviewed using GIS software: ArcMap (v10.1, ArcGIS, ESRI, 2012) and SAGA-GIS (v2.07, SAGA, 2012). ArcMap was used to identify points of overlap between the transportation lines (either railroad or highway) and county border lines, and these data were ported to a 
flat file for parsing using Perl. Effectively, an edge was formed between a county pair in the RR network when a railroad segment was detected crossing the border shared by the two counties (Figure 1). Collectively, the edge weight represented the total number of railroad crossings detected between the two counties. The same approach was used for highway crossings and the HWY network. Railroad and highway crossings were visually checked for accuracy using ArcGIS and/or SAGA. The graph data were ported into Python script running the NetworkX module (v 1.8; Hagberg et al. 2008; http://networkx.github.io/). This was used to convert the edge lists to graph objects for analysis.

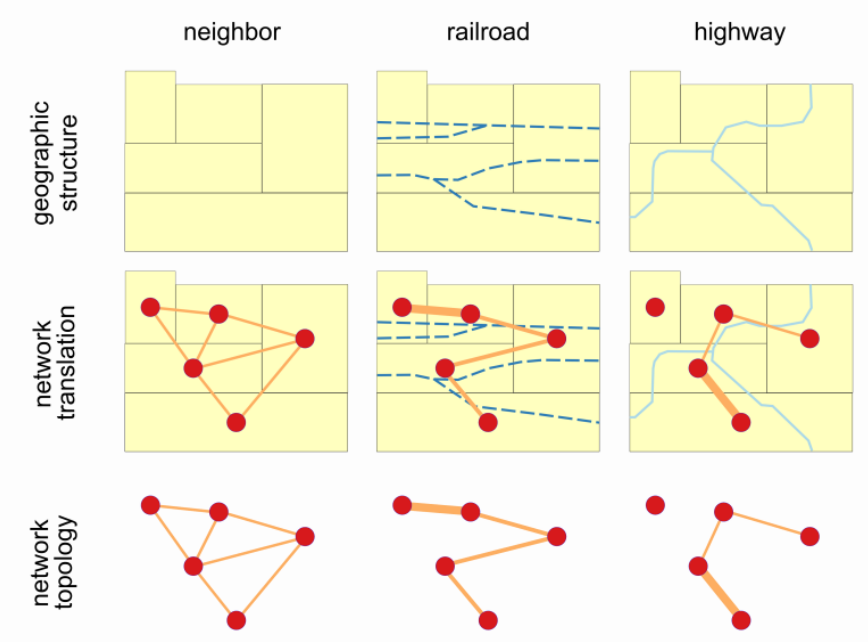

Figure 1. Method of network construction. The neighbor graph was an unweighted, simple subgraph of the geometric dual of the county polygon map. Further subgraphs were taken and weights added to represent the topology of transportation links in the railroad and highway networks.

The resulting graphs were equivalent to coarse-grained, G-space networks as described in (Wang et al., 2008). Under this construction, edge weights do not represent geodesic distances between counties; this was intentional in order to isolate topological properties of the transportation networks, as in Jiang and Claramunt (2004), and to assess small-world structure as originally defined by Watts and Strogatz (1998). 
By Kuratowski's Theorem (1930), a graph is planar if and only if it contains no subgraph homeomorphic to either of the complete graphs $\mathrm{K}_{5}$ or $\mathrm{K}_{3,3}$. But since this property is computationally intensive to verify for large graphs, we prove that our graphs, constructed as described herein, are planar. Briefly, as a null model, we produced a simple, non-transportation planar network (neighbor network, Figure 1) wherein nodes represented the 3,221 counties or county-equivalents in the contiguous United States, and an unweighted edge was formed between two counties if they shared a border. This network was essentially the geometric dual graph of the original polygon county map (Nishizeki et al., 2008), except we excluded multiple edges, loops, and the node representing the external, unbounded polygon. Since the dual graph of any planar graph is itself planar, and a subgraph of a planar graph is itself planar, our neighbor graph was planar. Steps in the transformation of the neighbor network into the two transportation networks included: (1) removal of edges where there was no transportation link crossing a border, (2) assignment of a non-zero weight to edges that represented the number of railroad or highway crossings between the respective counties, and (3) extraction of the largest connected component for analysis. The planar status was not modified by these steps of taking subgraphs or of adding weights to already existing edges, so our transportation networks were planar as well.

\subsection{Network analyses}

Networks were analyzed using script written in Python/NetworkX, with calculations of standard graph metrics performed as described in the manual. The significance of small-world structure was judged using Python script implementing Monte Carlo randomizations and the metric described in Humphries et al. (2006). All three networks were analyzed as weighted graphs, though all of the weights in the neighbor graph (non-transportation network) were 1.0, rendering it effectively an unweighted graph.

\subsubsection{Analysis of weighted graph metrics}

Aldrich et al. page 9 
Weighted node degree $\left(k_{i}\right)$, was calculated according to Barrat et al. (2004) where $a_{i j}=1$ if there is an edge between nodes $i$ and $j$, and $a_{i j}=0$ otherwise, and $w_{i j}$ is the weight of that edge across $N$ nodes of the graph:

$$
k_{i}=\sum_{j=1}^{N} a_{i j} w_{i j}
$$

Weighted clustering coefficient $\left(C_{i}\right)$ was calculated according to Onnela et al. (2005) where $k_{i}$ is the degree of node $i$, and $w_{i j}, w_{j k}$, and $w_{k i}$ the weights of the triangle that includes node $i$ and its neighbors $j$ and $k$ :

$$
C_{i}=\frac{2}{k_{i}\left(k_{i}-1\right)} \sum_{j, k}^{N}\left(w_{i j} w_{j k} w_{k i}\right)^{1 / 3}
$$

The weighted characteristic path length $(L)$ for each network was calculated as the mean of all the shortest paths between all node pairs. For weighted graphs, the shortest paths are typically calculated using Dijkstra's algorithm (Dijkstra, 1959), though this assumes that weights are treated as resistances to flow, such that paths are chosen to minimize total path length (sum of weights traversed). Since weights in our transportation networks represented channel capacities (number of links between counties), not resistances, we inverted the edge weights $(1 / w)$ for these calculations of $L$.

\subsubsection{Statistical comparison of networks}

In order to determine if the three graphs differed with respect to graph metrics, we conducted one-way ANOVAs for the degree and clustering coefficients, separately. Post-hoc pairwise comparisons were made using a Bonferoni correction. 


\subsubsection{Small-world analysis and Monte Carlo randomizations}

A small-world network (Watts and Strogatz, 1998) has a relatively high clustering coefficient and a relatively small characteristic path length. In order to test if a network displayed significant small-world structure, we employed the small-world coefficient (Humphries et al., 2006):

$$
S=\frac{C / C_{R}}{L / L_{R}}
$$

where the weighted clustering coefficient $(C)$ was calculated for the observed data as described above, and the characteristic path length for the observed data $(L)$ was calculated using inverse weights $(1 / w)$ and Dijkstra's algorithm (Dijkstra, 1959). Then the data were randomized 100 times, and these replicates were used for the calculation of $C^{*}$ and $L^{*}$; the mean values of the randomizations served as $C_{R}$ and $L_{R}$, respectively. $S>1.0$ indicates significant small-world structure provided $C / C_{R}>1.0$, under the rationale that one has normalized the observed clustering and path length estimates by the possible values that could arise at random in the system.

We implemented two separate Monte Carlo randomization methods. Under global ER randomization, each edge with its weight was re-assigned to a random node pair. While this provides a maximal amount of mixing, it produces unrealistic results in a planar graph. As an alternative, we also performed the more conservative planar randomization in which weighted edges were allowed to form randomly between counties only if they shared a border; edge weights were randomly assigned from the pool of observed edge weights. Under both procedures, the same number of edges were formed as existed in the respective observed networks. 


\section{Results and Discussion}

The three transportation networks are shown in Figure 2. For visualization, each node was positioned according to latitude and longitude of the county centroid as determined by ArcMap GIS, though edge weights did not represent geodesic distances.

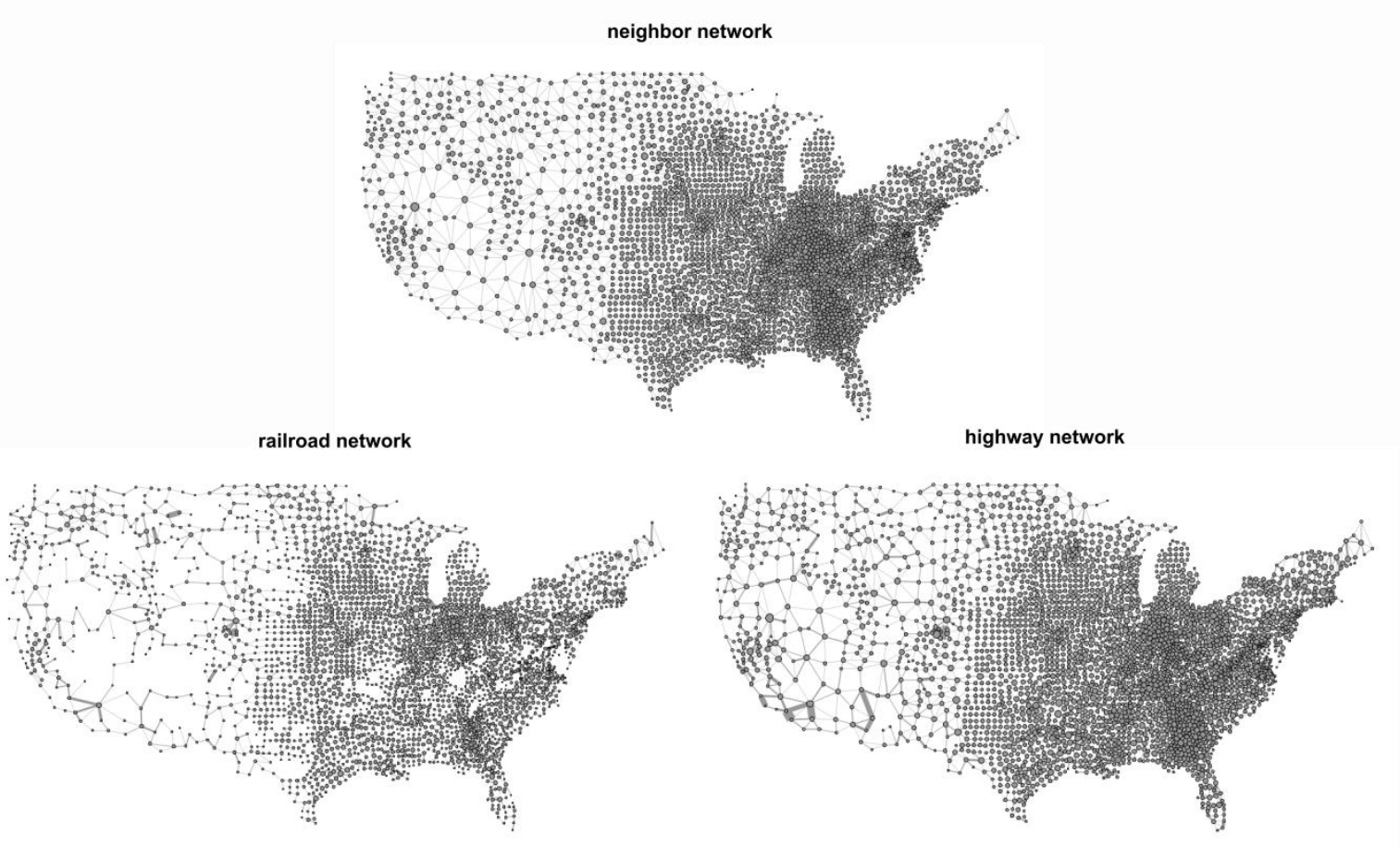

Figure 2. Largest connected components of three networks spanning the contiguous United States. Each node represents a county and weighted edges of the transportation networks represent the number of railroad or highway crossings between county pairs. The neighbor network is a simple, planar graph in which nodes also represent counties but edges are formed between counties only if they share a border, i.e., they do not represent transportation links. Node size represents weighted degree.

\subsection{Statistical comparison of networks}


Each network contained roughly 3,000 nodes representing the counties of the contiguous United States (Figure 2, Table 1). The two transportation networks were both subgraphs of the neighbor network, if one considers them all as unweighted graphs. Node count varied between graphs because not all counties were connected, and analyses were performed on the largest connected component of each graph. All three had roughly the same number of nodes in their largest connected component, with the railroad network at 93.4\% and the highway network at $99.7 \%$ of the nodes in the neighbor network $(n=3,105$; Table 1$)$. The differences among graphs were mostly due to the edge sets, where the railroad network had only $55.6 \%$ and the highway network $79.6 \%$ of the edges in the neighbor network $(e=8,685)$.

Table 1 Graph metrics for the three networks.

\begin{tabular}{|c|c|c|c|c|c|}
\hline Network & nodes & edges & $k$ & $C$ & $L$ \\
\hline Neighbor (NB) & 3,105 & 8,685 & $5.59^{\mathrm{a}}$ & $0.420^{\mathrm{a}}$ & 27.1 \\
\hline Railroad (RR) & 2,901 & 4,829 & $6.55^{b}$ & $0.0101^{\mathrm{b}}$ & 18.9 \\
\hline Highway (HWY) & 3,096 & 6,908 & $10.0^{\mathrm{c}}$ & $0.0133^{b}$ & 13.9 \\
\hline
\end{tabular}

nodes, number of county nodes in graph; edges, number of transportation links between county nodes; $k$, mean degree of county nodes; $C$, mean clustering coefficient of county nodes; $L$, characteristic path length for graph, calculated from inverse weights; All three networks were treated as weighted graphs, though in the neighbor network all edge weights were $1.0 ; a, b, c$ represent statistical equivalency categories under two separate ANOVA tests for node degree and clustering coefficient.

ANOVA tests showed that the three networks were significantly different for both weighted node degree $(p<0.0001)$ and weighted clustering coefficient $(p<0.0001$; Table 1). For pairwise comparisons, all three networks were different with respect to node degree, with the highway network exhibiting the most county crossings. For weighted clustering coefficient, we also found a significant difference 
between the three networks $(p<0.0001)$. However, this time the neighbor and highway networks were significantly different $(p<0.0001)$, and the neighbor and railroad networks were significantly different $(p$ $<0.0001)$, while the railroad and highway networks were statistically equivalent $(p=0.4)$. All comparisons were done using a Bonferroni correction.

As for characteristic path length, this was a global property of each graph and so an ANOVA was not performed. Characteristic path length was shorter in the transportation networks compared to the neighbor graph.

\subsection{Monte Carlo tests of small world structure}

\subsubsection{Global randomizations}

Both the railroad and highway networks displayed significant small-world structure relative to the ER

global randomizations (Table 2). In each case, the small-world coefficient was significant with $S>>1.0$. The second criterion of high clustering was also met, where the clustering coefficients were roughly 200fold higher in the observed transportation networks compared to global randomizations. These results agree with the general findings from other studies that small-worldness is a common feature of terrestrial transportation networks (Jiang and Claramunt, 2004; Latora and Marchiori, 2002, 2003; Li and Cai, 2007; Seaton and Hackett, 2004; Sen et al., 2003; Sienkiewicz and Hołyst, 2005; Wang et al., 2008). 
Table 2 Small world coefficients and tests against randomizations.

$\begin{array}{lllllllll}\text { Network } & C & C_{R} & C / C_{R} & L & L_{R} & L / L_{R} & S\end{array}$

\begin{tabular}{|c|c|c|c|c|c|c|c|}
\hline Neighbor (NB) & 0.420 & & & 27.1 & & & \\
\hline ER global randomization & & 0.00178 & 236 & & 4.86 & 5.58 & $42.4 *$ \\
\hline neighbor randomization & & 0.420 & 1.00 & & 27.1 & 1.00 & 1.00 \\
\hline Railroad (RR) & 0.0101 & & & 18.9 & & & \\
\hline ER global randomization & & 0.0000604 & 167 & & 4.19 & 4.51 & $37.1 *$ \\
\hline neighbor randomization & & 0.0124 & 0.815 & & 20.6 & 0.917 & 0.889 \\
\hline Highway (HWY) & 0.0133 & & & 13.9 & & & \\
\hline ER global randomization & & 0.0000607 & 219 & & 2.91 & 4.78 & $45.8 *$ \\
\hline neighbor randomization & & 0.0148 & 0.899 & & 13.8 & 1.01 & 0.895 \\
\hline
\end{tabular}

However, the overall traversability of the two transportation networks was less compared to the global randomizations $\left(L / L_{R}\right)$. Both of the characteristic path lengths for the observed networks were over 4-fold longer than that of the globally randomized networks: railroad (observed L, 18.9; global randomization $L_{R}$, 4.19; Table 2), highway (observed $L, 13.9$; global randomization $L_{R}, 2.91$ ). This runs counter to the general impression that small-world networks are easily traversed while still maintaining high local clustering. Apparently, this deficit in traversability is more than offset by the 200 -fold higher clustering of the transportation networks so that the small-world coefficient still registers as significant. This makes sense in that transportation networks are spatially constrained, two-dimensional systems in 
which it is difficult to establish long-distance shortcuts across the network, but there are ample opportunities for the formation of triangular relationships (Barthélemy, 2011).

Yet, this raises a question: Is the small-world architecture detected in transportation networks a result of the pattern of transportation links, or is it simply a manifestation of the planarity of the network? It is true that the observed characteristic path lengths were almost 2-fold shorter for the transportation networks (railroad $L, 18.9$; highway $L, 13.9)$ compared to the neighbor network $(L, 27.1)$. Yet, the clustering observed in the neighbor network was over 30-fold greater than either of the two transportation networks: neighbor $(C, 0.420)$, railroad $(C, 0.0101)$, highway $(C, 0.0133)$. This pattern suggests that the small-world architecture of transportation networks exists despite the pattern of transportation links, not because of it.

Such findings have a precedent. For example, one study (Jiang and Claramunt, 2004) looked at large urban street networks in Gavle (Sweden), Munich (Germany), and San Francisco (USA) and found small-world structure when compared to ER random graph theory expectations, i.e., a globally random model. Like our findings, they found that the observed characteristic path length $(L)$ was slightly larger than expectations for a similar-sized ER random graph $\left(L / L_{R}\right.$ : Gavle, 1.26; Munich, 1.48; San Francisco, 1.09), while the observed clustering coefficient was much larger than the ER random expectations $\left(C / C_{R}\right.$ : Gavle, 26.9; Munich, 35.8; San Francisco, 11.8). In our assessments of global randomizations, the average ratio of $L / L_{R}$ was 4.96 and $C / C_{R}$ was 207 .

Further evidence that small-world structure is independent of the pattern of transportation links came from the finding that the neighbor network displayed significant small-world structure when compared to global randomizations. The unweighted edges in this observed planar network were formed strictly among adjacent counties; there were no transportation links represented here. The small-world coefficient $(S, 42.4$, Table 2$)$ was slightly larger than that for the railroad network $(S, 37.1)$ and slightly smaller than that for the highway network $(S, 45.8)$. As for the second criterion of high clustering, the clustering coefficient was also roughly 200 -fold higher than the global randomizations. This, would suggest that the planarity of the network is the driver of its small-world structure.

Aldrich et al. page 16 


\subsubsection{Planar randomizations}

Our planar randomizations also indicated that terrestrial transportation systems, at least topologically, do not contribute much small-world structure beyond that already present in planar graphs. When edges were randomly assigned only to neighboring county pairs, all three networks failed to display significant smallworld structure. For the transportation networks, $S$ was less than one whereas $S$ was equal to one in the neighbor network. This latter result was due to the fact that spatially constrained randomizations of the unweighted neighbor network had no effect on topology since each county was fully connected to all of the counties that shared a border.

When we examined individually the clustering and traversability in the planar randomizations, these also indicated that transportation links added little small-world structure beyond that already present in planar randomized networks. In both transportation networks, the observed clustering was less than that in the constrained randomizations, i.e., there were fewer triangular relationships than expected by chance under these planar expectations. As for traversability, the highway network had a slightly longer characteristic path length compared to the planar randomizations $\left(L, 13.9 ; L_{R}, 13.8\right)$, while the railroad network was slightly more traversable than the planar randomizations $\left(L, 18.9 ; L_{R}, 20.6\right)$. Nevertheless, the low clustering of both transportation networks was not sufficient to register significance in the smallworld coefficient.

\section{Summary}

These results agree with the general finding that human terrestrial transportation networks have a smallworld architecture - however, only in the context of comparing the observed data with a globally randomized model. And here, the small-world structure owes mostly to exceptionally high clustering 
relative to the completely (globally) randomized condition; as for the other component of small-worldness (Watts and Strogatz, 1998), transportation networks actually are less easily traversed compared to the globally random networks. This finding reveals a general feature of transportation networks. The topologies of the observed railroad and highway networks have no more small-worldness than does a simple, planar, neighbor network or randomized planar networks. This indicates that the small-world signature that is detected under global randomizations is a general property of planar graphs, not necessarily a special property of transportation graphs. It should be noted that these findings are specifically relevant to small-worldness owing to the pattern or topology of linkages in a graph; the role played by geodesic length variation in the production of non-standard small-world architecture is another matter worth considering as others have already pointed out.

Acknowledgments We thank the US Department of Transportation, the Federal Highway Administration, the Federal Railroad Administration, and the US Census Bureau for use of their data. This work was supported in part by grants from the USDA Forest Service [04-JV-11242328-119, 05-JV11242343-057, and 06-JV-11242343-057 to PA and GG], Illinois Bord of Higher Education [05S001270001 to PA], and Benedictine University. The authors declare no conflict of interest. 


\section{References}

Barrat, A., Barthélemy, M., Pastor-Satorras, R., and Vespignani, A. (2004). The architecture of complex weighted networks. Proc. Natl. Acad. Sci. U. S. A. 101, 3747-3752.

Barthélemy, M. (2011). Spatial networks. Phys. Rep. 499, 1-101.

Blanchard, P., and Volchenkov, D. (2008). Mathematical Analysis of Urban Spatial Networks (Berlin: Springer).

Cardillo, A., Scellato, S., Latora, V., and Porta, S. (2006). Structural properties of planar graphs of urban street patterns. Phys. Rev. E 73, 066107.

Dijkstra, E.W. (1959). A note on two problems in connexion with graphs. Numer. Math. 1, 269-271.

Erdős, P., and Rényi, A. (1959). On random graphs. Publ. Math. 6, 290-297.

Erdős, P., and Rényi, A. (1960). On the evolution of random graphs. Publ. Math. Inst. Hung. Acad. Sci. 5, $17-61$.

Erdős, P., and Rényi, A. (1961). On the strength of connectedness of a random graph. Acta Math. Sci. Hung. 12, 261-267.

Floerl, O., Inglis, G.J., Dey, K., and Smith, A. (2009). The importance of transport hubs in stepping-stone invasions. J. Appl. Ecol. 46, 37-45.

Gastner, M.T., and Newman, M.E.J. (2006). The spatial structure of networks. Eur. Phys. J. B 49, 247252.

Harrison, S., Hohn, C., and Ratay, S. (2002). Distribution of Exotic Plants along Roads in a Peninsular Nature Reserve. Biol. Invasions 4, 425-430.

Hulme, P.E. (2009). Trade, transport and trouble: managing invasive species pathways in an era of globalization. J. Appl. Ecol. 46, 10-18.

Humphries, M.., Gurney, K., and Prescott, T.. (2006). The brainstem reticular formation is a small-world, not scale-free, network. Proc. R. Soc. B Biol. Sci. 273, 503-511.

Jiang, B., and Claramunt, C. (2004). Topological analysis of urban street networks. Environ. Plan. B Plan. Des. $31,151-162$.

Kalapala, V., Sanwalani, V., Clauset, A., and Moore, C. (2006). Scale invariance in road networks. Phys. Rev. E 73, 026130.

Kara, B.Y., and Verter, V. (2004). Designing a Road Network for Hazardous Materials Transportation. Transp. Sci. 38, 188-196.

Kim, B. (2004). Geographical Coarse Graining of Complex Networks. Phys. Rev. Lett. 93, 168701.

Kuratowski, K. (1930). Sur le problème des courbes gauches en topologie. Fund Math 15, 271-283. 
Latora, V., and Marchiori, M. (2002). Is the Boston subway a small-world network? Phys. A 314, 109113.

Latora, V., and Marchiori, M. (2003). Economic small-world behavior in weighted networks. Eur. Phys. J. B - Condens. Matter 32, 249-263.

Li, W., and Cai, X. (2007). Empirical analysis of a scale-free railway network in China. Phys. Stat. Mech. Its Appl. 382, 693-703.

Lin, J., and Ban, Y. (2013). Complex Network Topology of Transportation Systems. Transp. Rev. 33, $658-685$.

Masucci, A.P., Smith, D., Crooks, A., and Batty, M. (2009). Random planar graphs and the London street network. Eur. Phys. J. B 71, 259-271.

Metropolis, N., and Ulam, S. (1949). The Monte Carlo Method. J. Am. Stat. Assoc. 44, 335.

Meyerson, L.A., and Mooney, H.A. (2007). Invasive alien species in an era of globalization. Front. Ecol. Environ. 5, 199-208.

Morris, M. (2004). Network Epidemiology (Oxford University Press).

Newman, M.E.J. (2003). Random graphs as models of networks,. In Handbook of Graphs and Networks, (Berlin: Wiley-VCH),

Newman, M.E.J. (2010). Networks: An Introduction (Oxford: Oxford University Press).

Nishizeki, T., Chiba, N., and Mathematics (2008). Planar Graphs: Theory and Algorithms (Mineola, N.Y: Dover Publications).

Onnela, J.-P., Saramäki, J., Kertész, J., and Kaski, K. (2005). Intensity and coherence of motifs in weighted complex networks. Phys. Rev. E 71, 065103.

Perrings, C., Dehnen-Schmutz, K., Touza, J., and Williamson, M. (2005). How to manage biological invasions under globalization. Trends Ecol. Evol. 20, 212-215.

Pimentel, D., Zuniga, R., and Morrison, D. (2005). Update on the environmental and economic costs associated with alien-invasive species in the United States. Ecol. Econ. 52, 273-288.

Rodrigue, J.-P., Notteboom, T., and Shaw, J. (2013). The SAGE Handbook of Transport Studies (Thousand Oaks, CA: SAGE Publications Ltd).

Seaton, K.A., and Hackett, L.M. (2004). Stations, trains and small-world networks. Phys. Stat. Mech. Its Appl. 339, 635-644.

Sen, P., Dasgupta, S., Chatterjee, A., Sreeram, P., Mukherjee, G., and Manna, S. (2003). Small-world properties of the Indian railway network. Phys. Rev. E 67, 036106.

Sienkiewicz, J., and Hołyst, J. (2005). Statistical analysis of 22 public transport networks in Poland. Phys. Rev. E 72, 046127.

Song, C., Havlin, S., and Makse, H.A. (2005). Self-similarity of complex networks. Nature 433, 392-395. 
Travers, J., Milgram, S., Travers, J., and Milgram, S. (1969). An Experimental Study of the Small World Problem. Sociometry 32, 425-443.

Valverde, S., Cancho, R.F., and Solé, R.V. (2002). Scale-free networks from optimal design. Europhys. Lett. EPL 60, 512-517.

Wang, R., Tan, J.-X., Wang, X., Wang, D.-J., and Cai, X. (2008). Geographic coarse graining analysis of the railway network of China. Phys. Stat. Mech. Its Appl. 387, 5639-5646.

Watts, D.J., and Strogatz, S.H. (1998). Collective dynamics of "small-world" networks. Nature 393, 440442.

Xu, Z., and Sui, D.Z. (2007). Small-world characteristics on transportation networks: a perspective from network autocorrelation. J. Geogr. Syst. 9, 189-205. 